\title{
Sinusoplastía con balón en seno frontal: Experiencia en 8 pacientes
}

\author{
Sinuplasty balloon in frontal sinus: Experience in 8 patients
}

\author{
Rodolfo Nazar $\mathbf{S}^{1}$, Javiera Pardo J², Thomas Kühnel ${ }^{3}$, Daniel Simmen ${ }^{4}$.
}

\begin{abstract}
RESUMEN
Introducción: La sinusoplastía con balón es una técnica cada vez más usada en patología sinusal, ya que previene el trauma, conserva la anatomía, evita la instrumentación del seno frontal, preserva la mucosa del receso frontal, con un alto grado de seguridad. Dentro de sus principales indicaciones se encuentra la patología de seno frontal, como es el caso de sinusitis aislada, osteítis focalizada, sinusitis posoperatoria y barotrauma. Las controversias se relacionan con sus indicaciones limitadas, costo actual elevado y la ausencia de remoción de tejido.

objetivo: Determinar la efectividad de la sinusoplastía con balón en patología del seno frontal.

Material y método: Mediante estudio retrospectivo descriptivo se analizan 8 casos clínicos, antecedentes demográficos, indicación quirúrgica, técnica utilizada, seguimiento posoperatorio y complicaciones.

Resultados: Ocho pacientes, con edad promedio de 51,4 años. En la mayoría de los pacientes se utilizó balón más técnica quirúrgica endoscópica clásica (7/8). En todos se evidenció permeabilidad del seno frontal posoperatoria y al año de seguimiento. Ningún paciente tuvo complicaciones. El seguimiento promedio fue de 22,2 meses.

Conclusiones: La sinuplastía con balón es una técnica segura, siendo una herramienta más, disponible en cirugía nasosinusal. Es especialmente útil en dilatar el receso del seno frontal permitiendo su ventilación y drenaje. El conocimiento anatómico previo es esencial para su correcto uso.
\end{abstract}

Palabras clave: Sinusoplastía, balón, seno frontal.

\begin{abstract}
Introduction: Sinuplasty with balloon is a technology increasingly used in sinus pathology, avoiding trauma, and preserving anatomy. It avoids the instrumentation of frontal sinus, and can be realized even in ambulatory form, with high degree of safety. Main indications are the pathologies of frontal sinus, such as isolated sinusitis, focused osteitis, postoperatory sinusitis or barotrauma, where it allows the conservation of the

\footnotetext{
Médico Servicio de Otorrinolaringología, Hospital Clínico Universidad de Chile.

2 Médico Cirujano. Tesista de Magíster en Ciencias Biomédicas, mención Neurociencias, Universidad de Chile.

3 Médico Servicio de Otorrinolaringología. HNO Klinik Universität Regensburg.

4 Médico Servicio de Otorrinolaringología. ORL Zentrum, Zürich. Clínica de Otorrinolaringología. Hospital Clínico de la Universidad de Regensburg. Sección de Rinología. ORL Zentrum, Zürich.
} 
mucose of the frontal recess. Controversies relate to especific indications, cost and the absence of removal tissue.

Aim: Asses the effectiveness of balloon sinuplasty in pathology of the frontal sinus.

Material and method: Retrospective descriptive study analizing 8 clinical cases, demographic data, surgical indication, technology used, postoperatory follow-up and complications.

Results: Eight patients, average age 51,4 years. In the majority of the patients was used balloon and endoscopic surgical (7/8). In all cases technique was demonstrated postoperatory permeability of the frontal sinus. No one patient report complications, with an average follow-up of 22,2 months.

Conclusions: Balloon sinuplasty is a sure tool, and is a complementary instrument for nasosinusal surgery, being specially useful in dilatation and ventilation of the frontal recess. Anatomical previous knowledge is essential for its correct use.

Key words: Sinuplasty balloon, frontal sinus.

\section{INTRODUCCIÓN}

El manejo de la patología de las cavidades paranasales ha ido evolucionando en el tiempo desde las técnicas abiertas, pasando a las técnicas endoscópicas a partir de los años $80^{1-3}$. La cirugía endoscópica funcional es actualmente el método de elección para el manejo de la mayoría de las afecciones de las cavidades paranasales 4 .

Una nueva alternativa de tratamiento se ha incorporado recientemente: la dilatación de los drenajes de las cavidades paranasales mediante el uso de balones (sinuplastía con balón).

Las bases de la sinusoplastía con balón provienen de otras especialidades médicas, tales como cirugía cardiovascular, oftalmología y gastroenterología. Su aplicación en otorrinolaringología, específicamente en rinosinusología, se produce en el año 2005, mediante el uso de guía fluoroscópica 5 .

Los fundamentos que apoyan su uso en patología sinusal son la prevención del trauma, preservación de la anatomía, mantención de la mucosa indemne, especialmente en el receso frontal. Además posibilita tratamiento ambulatorio en la consulta y disminuiría los riesgos de complicaciones asociadas a la instrumentación de los senos paranasales, especialmente en patología seno frontal ${ }^{6}$.

En relación a la seguridad de la técnica ${ }^{5-11}$, actualmente existe vasta experiencia a nivel internacional, con una tasa de complicaciones de $0,01 \%$ por paciente (complicaciones mayores) ${ }^{12}$.
Existen algunas complicaciones menores como la ruptura del balón y que el dispositivo se desinfle lentamente.

Dentro de sus indicaciones se encuentra el manejo de la rinosinusitis crónica y aguda, el tratamiento del seno frontal con anatomía compleja y estrecha del receso del frontal, rinosinusitis aislada, especialmente de seno frontal y esfenoidal, ya sea aguda o posoperatoria, osteítis focalizada y barotrauma.

Si bien sus indicaciones específicas se encuentran claras, también existe consenso sobre situaciones donde no debe usarse, como es el caso de la poliposis nasosinusal difusa, pacientes con patología tumoral, presencia de cuerpo extraño 0 diagnóstico de bola fúngica (Tabla 1).

\section{Modo de empleo}

Esta técnica puede emplearse bajo anestesia local, ya sea en forma ambulatoria 0 en el caso de pacientes hospitalizados en unidades de cuidados intensivos. Además puede ser usada bajo anestesia general.

Se utiliza una guía de transiluminación o control fluoroscópico, que permite identificar el seno paranasal a tratar.

En cuanto a la técnica, ésta puede combinarse con las técnicas quirúrgicas endoscópicas clásicas (técnica híbrida) o en forma aislada, utilizando sólo el balón (Figura 1).

Es esencial que el procedimiento sea guiado por los hallazgos anatómicos evaluados previa- 
Tabla 1. Indicaciones y contraindicaciones en sinusoplastía con balón

\begin{tabular}{|ll|}
\hline Indicaciones & Contraindicaciones \\
\hline Rinosinusitis crónica y aguda & Poliposis difusa \\
Anatomía compleja y estrecha del receso del frontal & Tumor \\
Rinosinusitis aislada: frontal o esfenoidal & Cuerpo extraño \\
Sinusitis frontal aislada posoperatoria & Bola fúngica \\
Osteítis focalizada & Historia de trauma \\
Barotrauma & Keros III \\
\hline
\end{tabular}

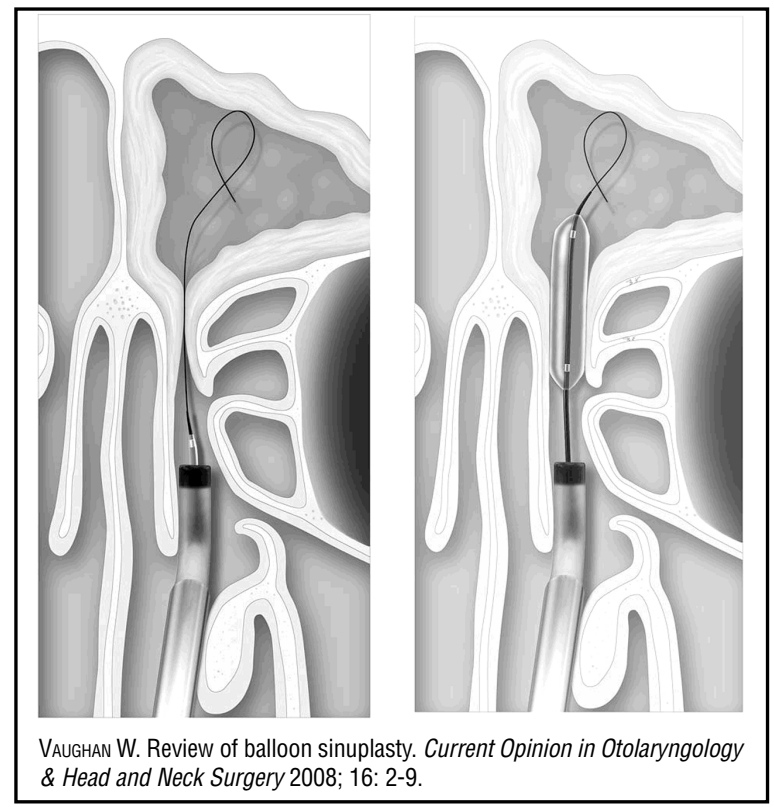

Figura 1. Sinusoplastía con balón. 1. Guía de transiluminación en el seno frontal. 2. Dilatación con balón del receso del seno frontal.

mente, mediante tomografía computarizada de cavidades paranasales, permitiendo incluso la reconstrucción tridimensional de la anatomía del paciente. Además la anatomía del receso del frontal, debe ser evaluada en forma precisa previo al procedimiento.

\section{Procedimiento}

En el caso de la técnica híbrida, inicialmente se realizan las etapas quirúrgicas necesarias de una cirugía endoscópica funcional, tales como uncinectomía-etmoidectomía anterior, para poste- riormente mediante dilatación con balón abrir el seno frontal con mínimo daño de la mucosa. El balón se infla con 12-16 atmósferas de presión ${ }^{13}$. Esta técnica es útil en identificar y dilatar el receso del frontal, considerándose un instrumento más en la mesa operatoria (Figura 2).

La guía de transiluminación actualmente ha reemplazado al control fluoroscópico, evitando el riesgo de exposición a radiación descrito con anterioridad ${ }^{10,14,15}$. Se describe un patrón clásico de transiluminación del seno frontal, el que es medial, para evitar su mal posición en una celdilla supraorbitaria y eventuales complicaciones orbitarias ${ }^{16}$ (Figura 3 ). 


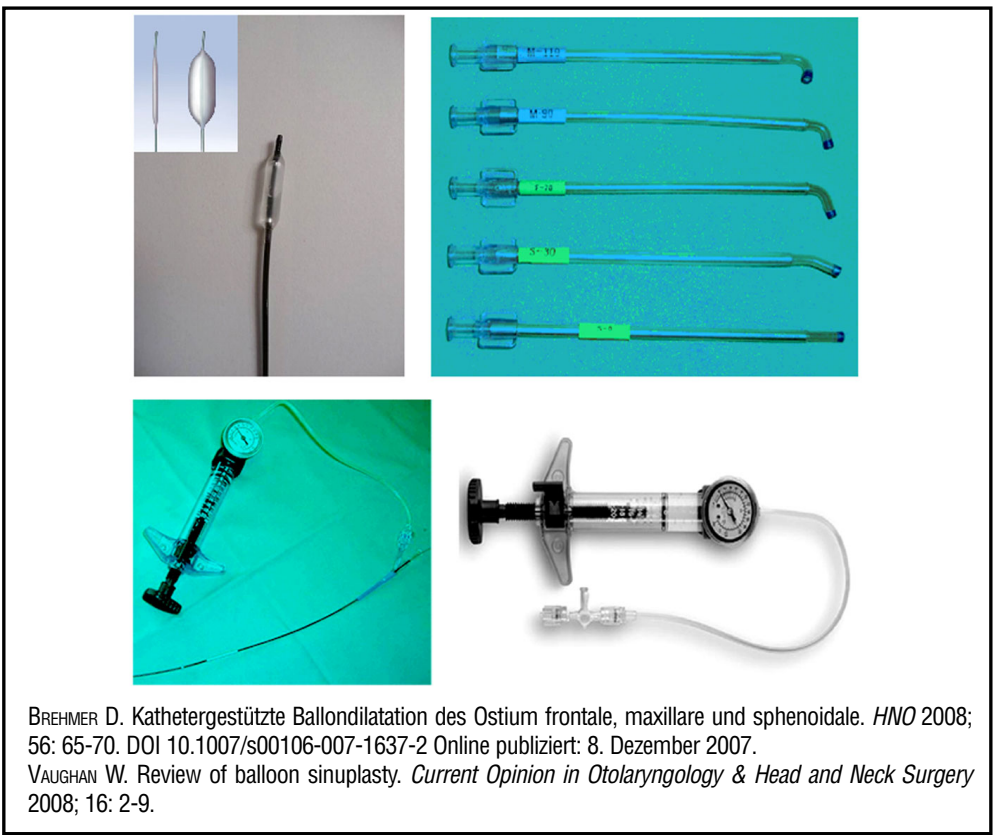

Figura 2. Balón y catéter utilizados durante el procedimiento. Se observan diferentes ángulos de catéter según seno a tratar.

\section{MATERIAL Y MÉTODO}

Estudio retrospectivo descriptivo realizado en el Hospital Clínico Universitario de Regensburg y en el ORL Zentrum de Zürich, entre los meses de junio de 2007 a julio de 2009. Se seleccionan un total de 8 pacientes, con un promedio de edad de 51,4 años, cuyos diagnósticos preoperatorios fueron: sinusitis frontal posoperatoria, barotrauma, rinosinusitis aguda recurrente, rinosinusitis crónica, osteítis frontal aislada y sinusitis frontal aislada. Todos los pacientes aceptaron el procedimiento mediante un consentimiento informado preoperatorio.

\section{RESULTADOS}

La técnica realizada fue híbrida (cirugía endoscópica funcional + balón) en 7 de los 8 pacientes y en un solo caso se realizó sinuplastía con balón aislada. El seno frontal fue la única cavidad tratada.

En todos los pacientes el procedimiento se realizó con anestesia general.

En 4 pacientes se trató el seno frontal en forma unilateral, y en 4 casos bilateral. En todos los

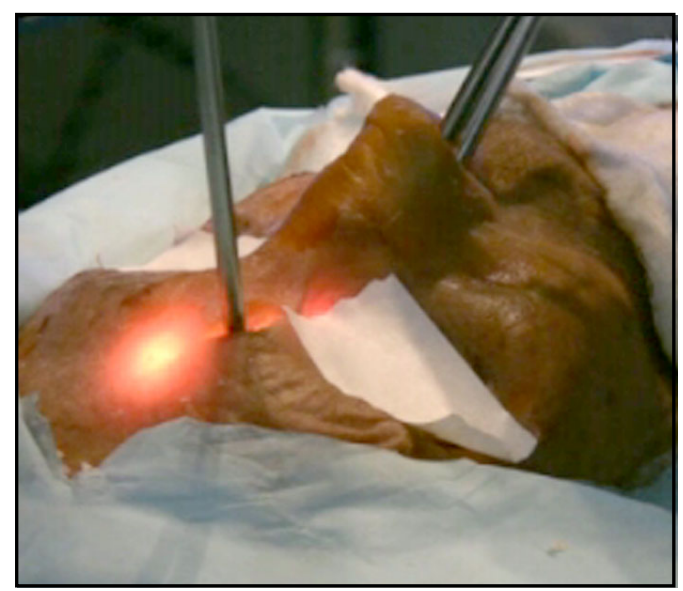

Figura 3. Uso de guía de transiluminación, en el seno frontal.

pacientes se usó guía de transiluminación. En un solo paciente se realizó además control radioscópico. El seno frontal se evidenció permeable mediante control endoscópico posoperatorio al año en todos los pacientes. El seguimiento posoperatorio fue en promedio de 22,2 meses con una variación de 12 a 38 meses. En los 8 pacientes 
operados no se registró ninguna complicación (Tabla 2).

La evaluación radiológica del paciente 2 muestra compromiso inflamatorio de ambos senos frontales (Figura 4). Durante el procedimiento se identifica el receso del frontal, que se canula con guía de transiluminación (Figura 5). El balón se infló en el receso frontal hasta 12 atmósferas de presión en dos oportunidades (Figura 6). Control endoscópico inmediato evidencia receso del frontal permeable (Figura 7 ).

\section{DISCUSIÓN}

La cirugía endoscópica es la técnica de elección para el tratamiento de patologías de cavidades para- nasales. La sinuplastía con balón es una reciente e innovadora alternativa de tratamiento en patología nasosinusal, que ha demostrado ser efectiva ${ }^{6}$. Permite restablecer el drenaje y la ventilación de los senos afectados ${ }^{17}$. Su uso estaría indicado en ciertas condiciones específicas (Tabla 1). La dilatación con balón es especialmente útil en cirugía de revisión y en sinusitis aislada del seno frontal, siendo una herramienta mínimamente invasiva ${ }^{9}$.

La revisión de literatura norteamericana nos revela que se utiliza para el tratamiento de afecciones de seno maxilar, esfenoides y frontal. En las grandes series clínicas se ve un promedio de tres senos tratados por paciente ${ }^{6}$.

En esta serie clínica inicial se presentan 8 casos de sinuplastía con balón exclusivamente en seno frontal (Tabla 2).

Tabla 2. Resultados

\begin{tabular}{|cclccc|}
\hline Paciente & $\begin{array}{c}\text { Edad } \\
\text { (años) }\end{array}$ & \multicolumn{1}{c}{ Indicación } & Técnica & $\begin{array}{c}\text { Seguimiento } \\
\text { (meses) }\end{array}$ & Complicaciones \\
\hline 1 & 58 & Sinusitis frontal posoperatoria & Híbrida & 38 & - \\
2 & 52 & Sinusitis frontal posoperatoria & Sólo balón & 16 & - \\
3 & 37 & Barotrauma. Rinosinusitis aguda & Híbrida & 12 & - \\
4 & 61 & Barotrauma. Rinosinusitis crónica & Híbrida & 34 & - \\
5 & 44 & Osté́tis frontal localizada. & Híbrida & 32 & - \\
& & Sinusitis frontal aislada & Híbrida & 16 & - \\
6 & 53 & Sinusitis frontal posoperatoria & Híbrida & 14 & - \\
7 & 46 & Rinosinusitis crónica & Híbrida & 16 & - \\
\hline
\end{tabular}

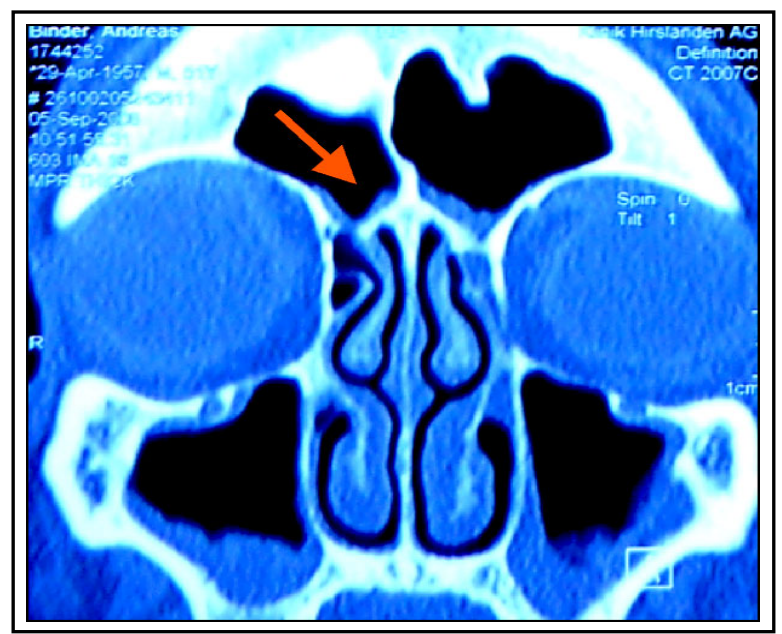

Figura 4. Tomografía computarizada de paciente №2. En corte coronal, se observa engrosamiento mucoso del receso del frontal bilateral con osteítis focalizada a izquierda. Además engrosamiento mucoso maxilar bilateral. 


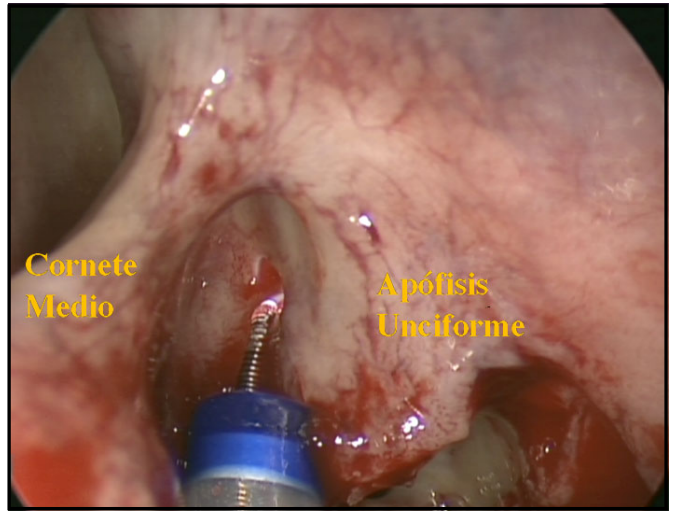

Figura 5. Con endoscopio de $45^{\circ}$ por fosa nasal izquierda se observa la guía de transiluminación introducida en el receso del frontal.

La cirugía endoscópica es una alternativa segura y eficiente para el tratamiento de las cavidades paranasales, sin embargo, el abordaje del seno frontal es todavía complejo con las actuales técnicas. El uso del balón sería una alternativa de tratamiento útil en patología frontal ${ }^{18}$.

Una de sus principales ventajas es la preservación de la mucosa con mínimo trauma, previniendo osteítis y reestenosis posoperatoria, fundamentalmente en el receso del frontal ${ }^{6,17}$. La tasa de reestenosis descrita es de $1,6 \%$ a las 24 semanas, en 5 de 307 pacientes $^{7}$. En nuestra serie el drenaje del seno frontal se evidenció permeable, en el control endoscópico al año de seguimiento en todos los pacientes.

Esta técnica evita la instrumentación del seno frontal, disminuyendo los eventuales riesgos $\mathrm{s}^{4,18}$. Otra ventaja del uso del balón es que permite la medicación intraseno de antibióticos y corticoides $^{19}$.

Dentro de las controversias que genera su uso, destacan sus limitadas indicaciones, costos actuales elevados, la ausencia de remoción de tejido y los riesgos de la exposición a radiación ${ }^{10,15,17}$. Actualmente esto último se ha solucionado con la técnica de transiluminación.

En el presente trabajo, la verificación de la introducción correcta del balón se realizó por medio de la sonda de transiluminación, en todos los pacientes. Un solo paciente requirió además de

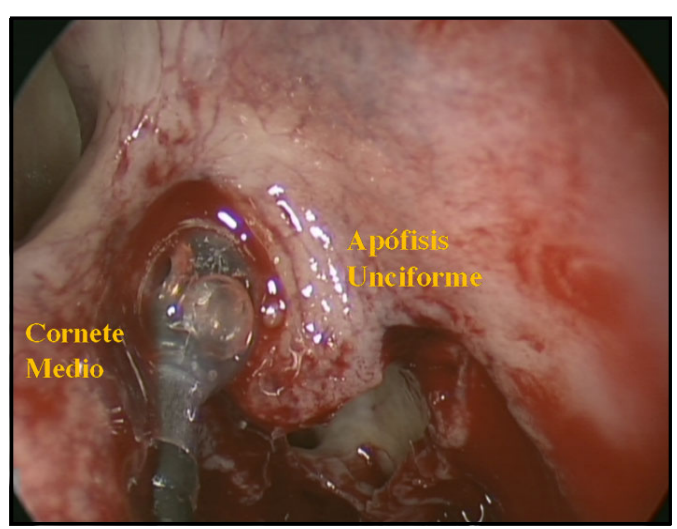

Figura 6. Con endoscopio de $45^{\circ}$ por fosa nasal izquierda, se observa balón inflado con 12 atmósferas de presión en receso del frontal. Se visualiza desplazamiento y fractura del remanente de apófisis unciforme.

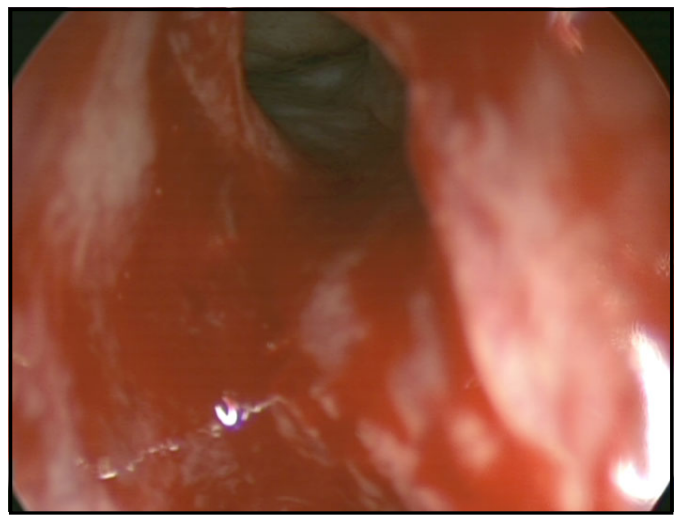

Figura 7. Con endoscopio de $45^{\circ}$ se observa receso del frontal izquierdo permeable posdilatación con balón.

la confirmación fluroscópica, al no tenerse un patrón claro de transiluminación frontal, debido a la dificultad para introducir la guía ${ }^{16}$ (Figura 2).

Las contraindicaciones de la sinuplastía aún no están claramente definidas, sin embargo algunas serían la sinusitis poliposa, historia de trauma y pacientes con una clasificación Keros tipo III en la tomografía computarizada de cavidades paranasales, debido al riesgo de lesión de la lamela lateral de la lámina cribosa ${ }^{17}$ (Tabla 1).

En relación a la técnica utilizada, en 7 de los 8 pacientes se realizó una técnica híbrida, partiendo el procedimiento con una cirugía endoscópica, 
previo a la dilatación con balón. Esto permitiría tener un mejor acceso a la zona del receso del frontal al realizar una resección previa de la apófisis unciforme. En un solo caso de sinusitis frontal posoperatoria se utilizó el balón en forma exclusiva.

El seguimiento clínico y endoscópico de nuestra casuística fue de 22,2 meses promedio, con una variación de 14 a 38 meses.

Se evidenció permeabilidad del receso del frontal en todos los casos en el control endoscópico al año. La tasa de permeabilidad descrita en la literatura es de $80,5 \%$ a las 24 semanas $^{7}$.

La literatura describe escasas complicaciones mayores. En una serie de 85.719 senos tratados se describen 3 complicaciones mayores (dos lesiones de lámina papirácea y una fístula de líquido cefalorraquídeo) con una tasa de complicaciones por paciente de $0,01 \%{ }^{12}$, por lo que en general se considera un procedimiento seguro. En el presente trabajo no evidenciamos complicaciones, en los 8 pacientes.

\section{CONCLUSIONES}

La sinuplastía con balón ofrece una alternativa de tratamiento disponible en cirugía endoscópica de cavidades paranasales. Permite restablecer el drenaje y la ventilación de los senos afectados, con mínimo trauma.

En nuestra casuística el balón se utilizó exclusivamente en el seno frontal, siendo útil en identificar el seno y dilatar el receso en forma segura.

La creciente experiencia internacional aporta nueva evidencia para definir indicaciones precisas, utilidad clínica y seguimiento posoperatorio.

\section{BIBLIOGRAFÍA}

1. Stammberger $H$, Posametz $W$. Functional endoscopic sinus surgery. Concept, indications and results of the Messerklinger technique. Eur Arch Otorhinolaryngol 1990; 247(2): 63-76.

2. Kennedy DW, Zinreich SJ, Rosenbaum AE, Johns ME. Functional endoscopic sinus surgery. Theory and diagnostic evaluation. Arch Otolaryngol 1985; 111(9): 576-82.
3. Draf W, Weber R. Endonasal micro-endoscopic pansinusoperation in chronic sinusitis. I. Indications and operation technique. Am J Otolaryngol 1993; 14(6): 394-8.

4. Vaughan W. Review of balloon sinuplasty. Current Opinion in Otolaryngology \& Head and Neck Surgery 2008; 16: 2-9.

5. Bolger WE, Vaughan WC. Catheter-based dilation of the sinus ostia: initial safety and feasibility analysis in a cadaver model. $A m \mathrm{~J}$ Rhinol 2006; 20(3): 290-4.

6. Levine HL, Sertich AP $2^{\mathrm{ND}}$, Hoisington DR, Weiss RL, Pritikin J; Patient Registry Study Group. Multicenter registry of balloon catheter sinusotomy outcomes for 1,036 patients. Ann Otol Rhinol Laryngol 2008; 117(4): 263-70.

7. Bolger W, Brown C, Church C. Safety and outcomes of balloon catheter sinusotomy: $A$ multicenter 24-week analysis in 115 patients. Otolaryngology-Head and Neck Surgery 2007; 137: 10-20.

8. Kuhn F, Church C, Goldberg A. Balloon catheter sinusotomy: One-year follow-up-Outcomes and role in functional endoscopic sinus surgery. Otolaryngology-Head and Neck Surgery 2008; 139: S27-S37.

9. Weiss R, Church C, Kuhn F, Levine H, Sillers M, VAUGHAN W. Long-term outcome analysis of balloon catheter sinusotomy: Two-year followup. Otolaryngology-Head and Neck Surgery 2008; 139: S38-S46.

10. Church C, Kuhn F, Mikhall J, Vaughan W, Weiss R. Patient and surgeon radiation exposure in balloon catheter sinus ostial dilation. Otolaryngology-Head and Neck Surgery 2008; 138: 187-91.

11. Ramadan HH. Safety and feasibility of balloon sinuplasty for treatment of chronic rhinosinusitis in children. Ann Otol Rhinol Laryngol 2009; 118(3): 161-5.

12. Melroy CT. The balloon dilating catheter as an instrument in sinus surgery. Otolaryngol Head Neck Surg 2008; 139(3 Suppl 3): S23-6.

13. http://www.dupagemedicalgroup.com/ otolaryngology_ent/

14. Chandra R. Estimate of radiation dose to the lens in balloon sinuplasty. OtolaryngologyHead and Neck Surgery 2007; 137: 953-5. 
15. Albritton F, Levine H, Smith J. Surgeon radiation exposure in ESS with balloon catheters. Otolaryngology-Head and Neck Surgery 2009; 140: 834-40.

16. Friedman M, Wilson M. Illumination Guided Balloon Sinuplasty. Laryngoscope 2009; 119: 1399-402.

17. BreHMER D. Kathetergestützte Ballondilatation des Ostium frontale, maxillare und sphenoidale. HNO 2008; 56: 65-70. DOI 10.1007/s00106007-1637-2 Online publiziert: 8. Dezember 2007.

18. https://www.accessdata.fda.gov.

19. LANZA DC, KenNedy DW. Balloon sinuplasty: not ready for prime time. Ann Otol Rhinol Laryngol 2006; 115(10): 789-90; discussion 791-2. 